\title{
Suppression of A549 lung cancer cell migration by precursor let-7g microRNA
}

\author{
SUNGJIN PARK ${ }^{1}$, ARASH MINAI-TEHRANI ${ }^{1}$, CHENG-XIONG XU ${ }^{1}$, SEUNG-HEE CHANG ${ }^{1}$, MIN-AH WOO ${ }^{1,2}$, \\ MI-SUK NOH ${ }^{1,2}$, EUN-SUN LEE ${ }^{1}$, HWANG-TAE LIM ${ }^{1,2}$, GIL-HWAN AN ${ }^{3}$, KEE-HO LEE ${ }^{4}$, \\ HA-JUNG SUNG ${ }^{5}$, GEORGE R. BECK $\mathrm{Jr}^{6}$ and MYUNG-HAING CHO ${ }^{1,2}$
}

\begin{abstract}
${ }^{1}$ Laboratory of Toxicology, College of Veterinary Medicine, ${ }^{2}$ Department of Nano Fusion Technology, Graduate School of Convergence Science and Technology, Seoul National University, Seoul; ${ }^{3}$ Department of Food Science and Technology, College of Agriculture and Life Sciences, Chungnam National University, Daejeon; ${ }^{4}$ Laboratory of Molecular Oncology, Korea Institute of Radiological and Medical Sciences, Seoul; ${ }^{5}$ Institute of Fusion Technology, Hoseo University, Chungnam, Korea; ${ }^{6}$ Division of Endocrinology, Metabolism and Lipids,
\end{abstract} Emory University School of Medicine, Atlanta, GA, USA

Received June 22, 2010; Accepted September 14, 2010

DOI: $10.3892 / \mathrm{mmr} .2010 .373$

\begin{abstract}
Let-7g miRNAs, short non-coding RNAs approximately 21 nucleotides long, repress protein translation by binding to the 3'UTR of target mRNAs. Aberrant expression of let-7g is associated with the poor prognosis of lung cancer patients. Compared to normal lung cells, let-7g expression is absent in non-small cell lung cancer (NSCLC) cells. Furthermore, K-Ras and HMGA2 are well known as targets of let-7g. In this study, we evaluated the potential role of precursor (pre)-let-7g in lung cancer cell metastasis, focusing on the two targets of let-7g, HMGA2 and K-Ras. We found that pre-let-7g inhibited the migration of A549 lung cancer cells through HMGA2-mediated E2F1 down-regulation. Thus, our results suggest that pre-let- $7 \mathrm{~g}$ could be used as a suitable target for the suppression of lung cancer cell migration.
\end{abstract}

\section{Introduction}

MicroRNAs (miRNAs) are a class of short non-coding RNAs approximately 21 nucleotides long. They are converted to mature forms of miRNAs through stepwise processing, including the generation of 70 -nucleotide precursor (pre)-miRNAs. These pre-miRNAs have a characteristic hairpin structure from the longer nascent transcripts (primary miRNA), and are converted into a mature form by Dicer-mediated processing. Mature miRNAs regulate gene expression as translational repressors through highly conserved base-pairing to target mRNAs,

Correspondence to: Professor Myung-Haing Cho, Laboratory of Toxicology, College of Veterinary Medicine, Seoul National University, Seoul 151-742, Korea

E-mail: mchotox@snu.ac.kr

Key words: let-7g, microRNA, lung cancer cell migration typically at sites located in the 3'UTR region (1-3). In humans, thousands of miRNAs have been discovered (4), and miRNAs are predicted to control the activity of approximately $30 \%$ of all protein-coding genes. Individual miRNAs can target diverse mRNAs, and an individual mRNA can be regulated by several miRNAs; therefore, relatively few miRNAs can alter a large number of proteins $(5,6)$.

In particular, the let-7 miRNA family has been proposed to function in tumor suppression (7). There are at least 9 individual members of the let-7 family in mammals, and several let-7 genes are located in regions frequently deleted in human cancer (8). Moreover, let-7 expression was found to be reduced in a subset of non-small cell lung cancer (NSCLC) patients, and this reduction was highly correlated with poor prognosis (2).

Since the initial identification of K-Ras as a direct target of let-7 miRNA, several computational algorithms have identified HMGA2 as a convincing target of let-7 miRNA $(7,9,10)$. However, the consequences of let-7g targeting of both HMGA2 and K-Ras have not been investigated. In this study, we evaluated the potential role of pre-let-7g miRNA in lung cancer cell metastasis, focusing on the two targets of let-7g, HMGA2 and K-Ras. Here, we report that pre-let- $7 \mathrm{~g}$ inhibited migration of A549 lung cancer cells through HMGA2-mediated E2F1 down-regulation. Our results suggest that let-7g may be used as a good target for suppression of lung cancer cell migration.

\section{Materials and methods}

Reagents, plasmids and antibodies. Penicillin/streptomycin was purchased from GibcoBRL (Grand Island, NY, USA). Mirus Bio TransIT-LT1 transfection reagent and the siXpress ${ }^{\mathrm{TM}}$ expression vector were purchased from Mirus Bio (Madison, WI, USA). Mitomycin C and 4',6'-diamidino2-phenylindole (DAPI) were purchased from Sigma-Aldrich (St. Louis, MO, USA). Fluorescein isothiocyanate (FITC)conjugated AffiniPure goat anti-rabbit $\operatorname{IgG}$ was purchased 
from Jackson ImmunoResearch Laboratories (West Grove, PA, USA). The bicistronic construct, pcDNA-fLUC pol IRES-rLUC, was donated by Dr Gram (Novartis Pharma AG, Basel, Switzerland). Pre-let-7g was inserted into the siXpress expression vector. K-Ras siRNA, HMGA2 (HMGI-C) siRNA, siRNA transfection reagent, anti-K-Ras, anti-p53, anti-E2F1, and anti-eIF4E-BP1 antibodies were obtained from Santa Cruz Biotechnology (Santa Cruz, CA, USA). The antibody against HMGA2 was purchased from Biocheck (Foster City, CA, USA).

Cell culture and transfection. Human lung cell lines (A549, NL20, H1703, H520, H460 and H226) were originally obtained from ATCC. Cells were incubated in RPMI-1640 medium supplemented with 10\% FBS and penicillin/streptomycin. Cells were transiently transfected using Mirus Bio TransIT-LT1 transfection reagent according to the manufacturer's instructions.

Luciferase assay. For the luciferase assay, luciferase activities in the cell lysates were measured according to the manufacturer's instructions. Briefly, after transfection, cells were collected in passive lysis buffer (Promega, Madison, WI, USA). Cell lysates were centrifuged for $1 \mathrm{~min}$ at 13,000 rpm at $4^{\circ} \mathrm{C}$, and the supernatant was centrifuged for an additional $1 \mathrm{~min}$ at $13,000 \mathrm{rpm}$ at $4^{\circ} \mathrm{C}$. Firefly luciferase and renilla luciferase activities were measured using a dual luciferase assay kit (Promega).

Western blot analysis. Cells were grown in $75-\mathrm{cm}^{2}$ culture flasks. Controls were treated with transfection reagent, empty vector plasmids, or pre-let-7g plasmids for $48 \mathrm{~h}$. For extraction of total proteins, cells were lysed and centrifuged at $13,000 \mathrm{rpm}$ for $1 \mathrm{~min}$, and the supernatant was used for Western blotting. Protein concentration was measured using the BCA kit (Pierce, Rockfort, IL, USA), and $25 \mu \mathrm{g}$ of protein was separated on SDS-PAGE and transferred to nitrocellulose membranes (Amersham Pharmacia, Cambridge, UK). The membranes were blocked for $1 \mathrm{~h}$ in Tris-buffered saline and Tween-20 containing 5\% skim milk, and immunoblotting was performed by incubation overnight at $4^{\circ} \mathrm{C}$, and then with secondary antibodies conjugated to horseradish peroxide for $2 \mathrm{~h}$ at room temperature or overnight at $4^{\circ} \mathrm{C}$. After washing, the bands of interest were analyzed by the luminescent image analyzer LAS-3000 (Fujifilm, Tokyo, Japan), and quantification of Western blot analysis was performed using the Multi Gauge version 2.02 program (Fujifilm).

Immunocytochemistry. Cells were grown on cover slips in 2-well plates and transfected with pre-let-7g plasmids. After $48 \mathrm{~h}$, cells were rinsed twice with phosphate-buffered saline (PBS), fixed with $4 \%$ paraformaldehyde for $20 \mathrm{~min}$, and then permeabilized with $0.3 \%$ Triton X-100 for $10 \mathrm{~min}$. Cells were washed three times with PBS and treated with $3.7 \%$ bovine serum albumin (BSA) for $1 \mathrm{~h}$, followed by incubation with the anti-E2F1 antibody (1:1000 dilution) in 3.7\% BSA for $24 \mathrm{~h}$ at $4^{\circ} \mathrm{C}$. The primary antibody was removed from the cells, which were then washed three times with PBS and incubated with FITC-conjugated secondary antibodies (1:50) for $1 \mathrm{~h}$ at room temperature. Cells were counter-stained with DAPI for $30 \mathrm{~min}$

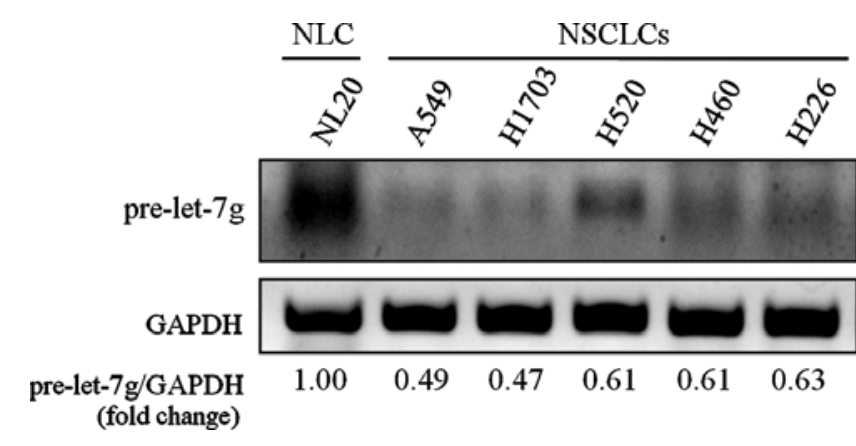

Figure 1. Deficiency of pre-let-7g in non-small cell lung cancer cell lines. Semi-qRT-PCR of expressed pre-let-7g in lung cancer cell lines compared with a normal lung cell line. Quantification (bottom) of expression data for normalized GAPDH. pre-let-7g, precursor let-7g; NLC, normal lung cells; NSCLCs, non-small cell lung cancer cells.

before visualization under a confocal microscope (Carl Zeiss, Jena, Germany).

RNA extraction and reverse transcription PCR. Pre-let-7g cDNA was synthesized from total RNA using specific primers as described in a previous study (11). Briefly, total RNA was extracted from $2 \times 10^{6}$ cells using TRIzol (Invitrogen, Carlsbad, CA, USA) according to the manufacturer's protocol. Total RNA was extracted from lung cancer cell lines using TRIzol and analyzed using the One-Step RT-PCR premix kit (Intron Biotechnology, Seongnam, Korea). Gene-specific primers included a mixture of $0.5 \mathrm{pM}$ each of the antisense primers to pre-let-7g and GAPDH. RT-PCR reaction of samples was performed according to the manufacturer's protocol.

Monolayer wound healing assay. In order to block cell proliferation, confluent A549 monolayers were scratched with a pipette tip, with a $30 \mathrm{~min}$ pre-incubation in the presence of the DNA synthesis inhibitor mitomycin C $(3 \mu \mathrm{g} / \mathrm{ml})$. Wound areas were observed using phase contrast microscopy on an inverted microscope. Images of the same areas were captured at regular intervals over the course of 0-96 h. The cell migration area was measured using Paint.Net v3.10 free software.

Flow cytometry for cell cycle analysis. After treatment, all of the cells were harvested by trypsinization, washed with icecold PBS and fixed in 70\% ice-cold ethanol diluted in PBS. Approximately $1 \times 10^{5}$ cells per tube were incubated in PBS containing $20 \mu \mathrm{g} / \mathrm{ml}$ RNase and $100 \mu \mathrm{g} / \mathrm{ml}$ propidium iodide at room temperature for 5-10 min before flow cytometric analysis. Cell cycle distribution was determined using a Jasco FP-6500 spectrofluorometer (Jasco Inc., Tokyo, Japan).

Statistical analysis. Data were analyzed using the Student's t-test (GraphPad software, San Diego, CA, USA). A P-value $<0.05$ was considered statistically significant.

\section{Results}

Pre-let-7g expression is absent in human lung cancer cells compared with normal lung cells. Deficiency of mature let-7 miRNA is known to be associated with poor prognosis in lung 
A

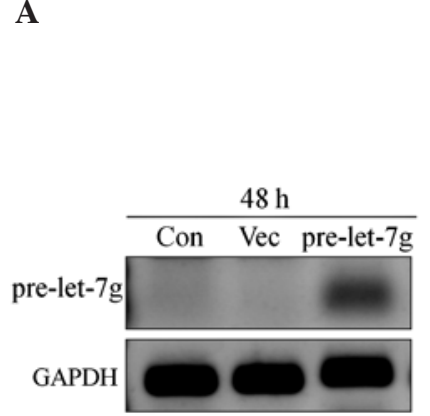

C
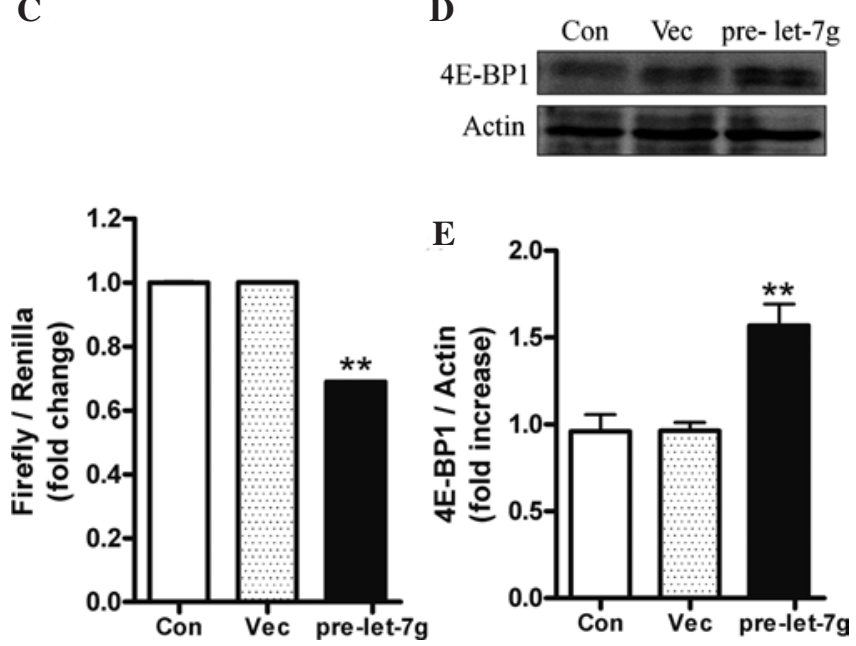

Figure 2. Suppression of cap-dependent protein translation by pre-let-7g. (A) Representative semi-qRT-PCR analysis demonstrating significant induction of pre-let-7g in A549 cells transfected with the pre-let-7g construct compared with the A549 control cells. (B) Quantification of expression data shown in A. (C) Relative ratio of firefly luciferase activity (cap-dependent promoter activity) normalized by renilla luciferase activity (pcDNA-firefly Luc/IRES-renilla Luc) in A549 cells transfected with the pre-let-7g construct compared with A549 control cells. (D) Representative Western blot analysis demonstrating a significant induction of 4E-BP1 in the A549 cells transfected with pre-let-7g compared with the A549 control cells. (E) Quantification of expression data shown in D. All samples were incubated for $48 \mathrm{~h}$ after transfection as shown in this figure. Con, control; Vec, vector control. Data represent the mean \pm SEM $(\mathrm{n}=3) .{ }^{* * *} \mathrm{P}<0.01$, statistically significant compared to the corresponding control.
A

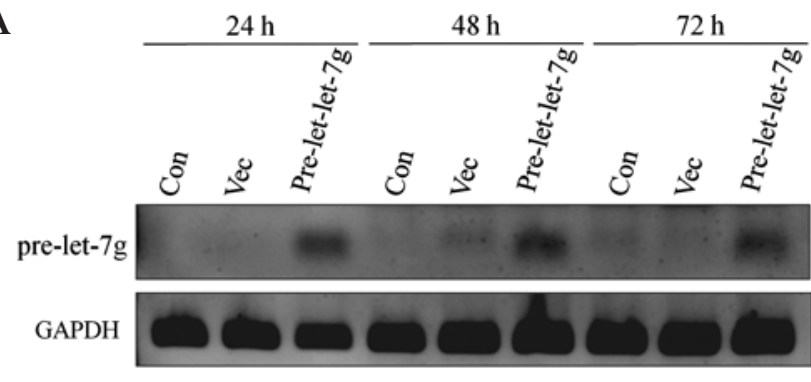

B
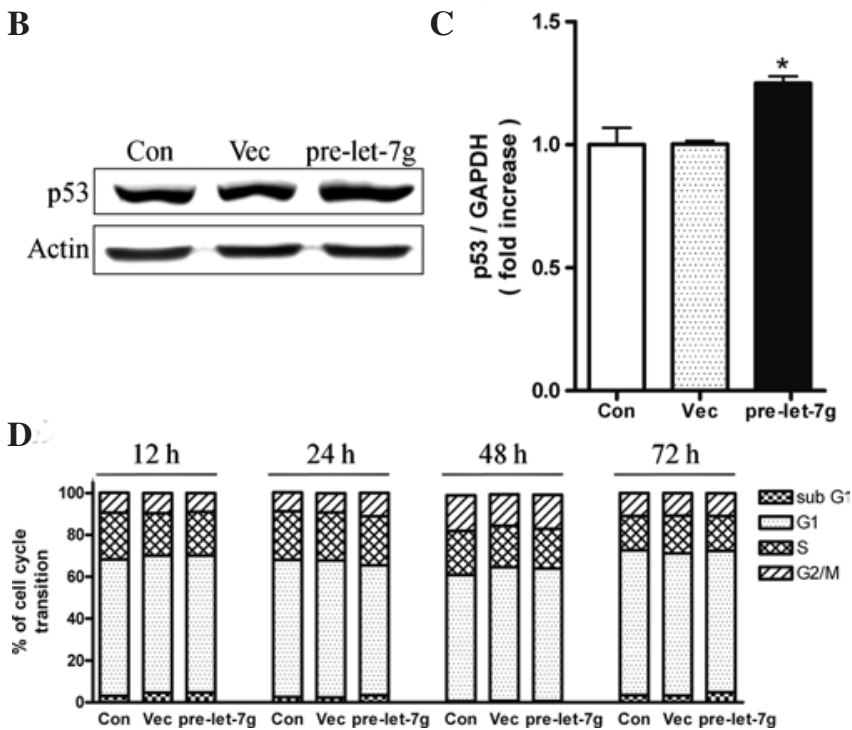

Figure 3. Continuous expression of pre-let-7g. (A) Representative semiqRT-PCR analysis demonstrating a marked induction of pre-let-7g in A549 cells incubated for 24,48 and $72 \mathrm{~h}$ after transfection with the pre-let- $7 \mathrm{~g}$ construct compared with the A549 control cells. (B) Representative Western blot analysis demonstrating a significant increase of p53 in the A549 cells transfected with pre-let-7g compared with the A549 control cells. (C) Quantification of expression data shown in B. (D) Flow cytometric analysis of the A549 cells incubated for 12, 24, 48 and $72 \mathrm{~h}$ after transfection with pre-let-7g compared with the A549 control cells. No significant differences were observed in the rate of cell cycle arrest by pre-let-7g. Con, control; Vec, vector control. Data represent the mean $\pm \operatorname{SEM}(n=3) .{ }^{*} \mathrm{P}<0.05$, statistically significant compared to the corresponding control. cancer patients (2). Precursor microRNA, 70 nucleotides long, can be quantified by PCR analysis. In order to investigate the expression level of pre-let-7g in several lung cancer cell lines (A549, H1703, H520, H460, H226), we extracted total RNAs and performed RT-PCR. Compared to the normal lung cells (NLCs), NL20, our results clearly demonstrated low expression of pre-let-7g in NSCLC (Fig. 1). In this regard, we chose A549 cells for further study.

Induction of pre-let- $7 \mathrm{~g}$ represses cap-dependent protein translation in A549 cells. Overexpression of pre-let-7g in A549 cells was confirmed by semi-qRT-PCR analysis. Our results showed that the pre-let-7g expression level was significantly increased after $48 \mathrm{~h}$ (Fig. 2A and B). miRNAs initiate the cap-dependent protein translation repression step by binding to complementary sequences in the 3'UTR of their target mRNAs (3). To investigate the functional roles of pre-let-7g miRNA, A549 cells were co-transfected with pre-let-7g and bicistronic constructs containing a firefly reporter protein driven by a cap-dependent sequence and a renilla reporter protein driven by an internal ribosome entry site (IRES), where the ratio of firefly/renilla luciferase represents cap-dependent protein translation activity. Pre-let-7g reduced firefly luciferase activity by approximately $30 \%$ compared to the control (Fig. 2C). These results indicate that pre-let-7g represses cap-dependent protein translation. We also performed Western blot analysis to investigate the protein level of eIF4E binding protein 1 (4E-BP1), known as cap-dependent translation inhibitor. Our results showed that pre-let-7g increased the protein level of 4E-BP1 (Fig. 2D), suggesting that pre-let-7g inhibits protein translation through cap-dependent protein translation repressor 4E-BP1.

Inducible pre-let-7g retains level of expression. SemiqRT-PCR was performed in order to determine the expression of pre-let-7g at 24,48 and $72 \mathrm{~h}$. Our results showed that prelet-7g expression increased as a function of time (Fig. 3A). Recent studies have shown the relationship between miRNA 

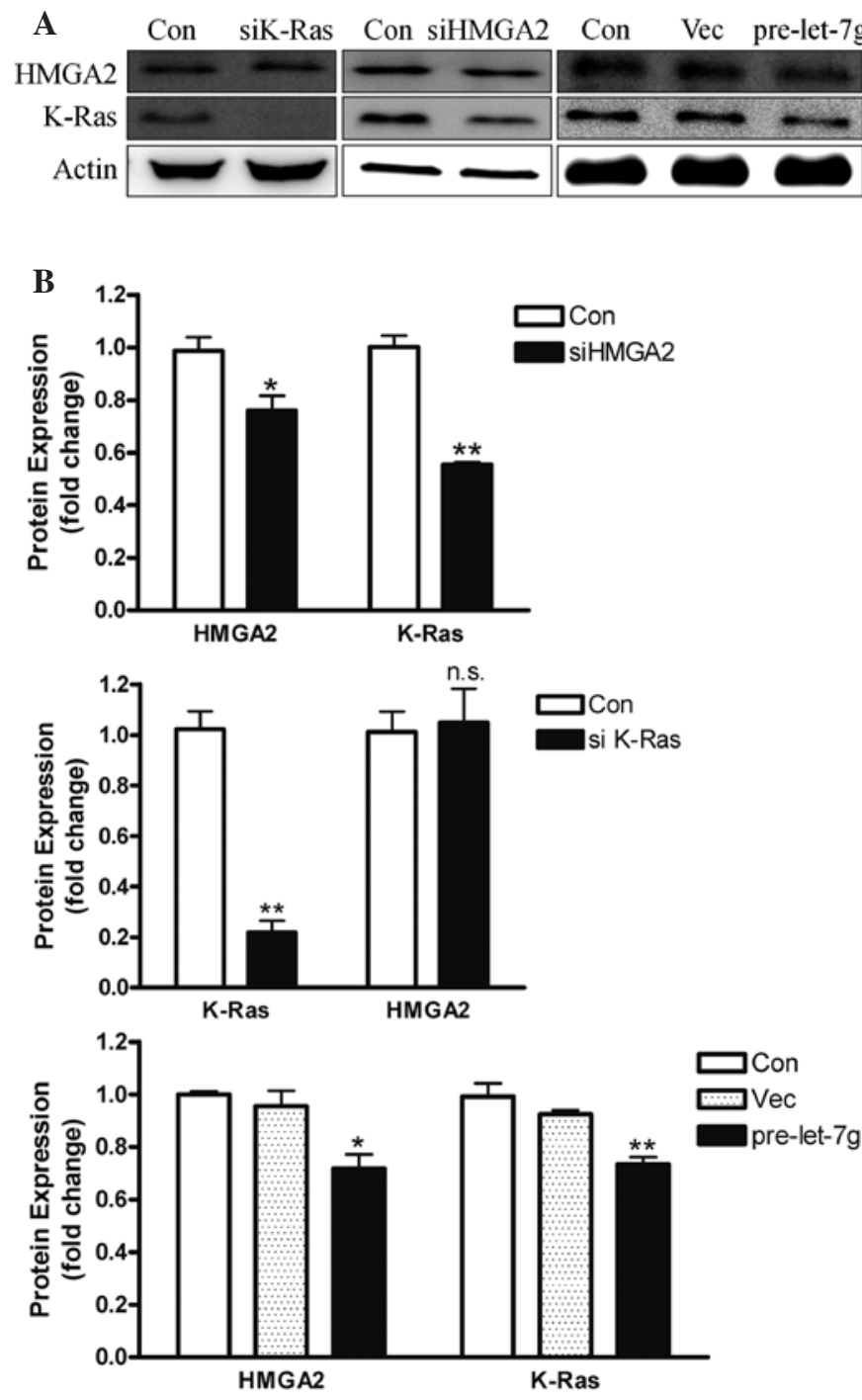

Figure 4. Pre-let-7g negatively regulates K-Ras and HMGA2. A549 cells were incubated for $48 \mathrm{~h}$ after transfection with pre-let-7g, siHMGA2 and siK-Ras. (A) Representative Western blot analysis of the expression levels of HMGA2 and K-Ras, compared with the A549 control cells. (B) Quantification of expression data shown in A. Con, control; Vec, vector control; siK-Ras, K-Ras siRNA; siHMGA2, HMGA2 siRNA. Data represent the mean \pm SEM $(n=3) .{ }^{*} \mathrm{P}<0.05$ or ${ }^{* *} \mathrm{P}<0.01$, statistically significant compared to the corresponding control.

and $\mathrm{p} 53$ in the regulation of miRNA $(12,13)$. We investigated p53 expression as a possible regulator of pre-let-7g. The results revealed that pre-let-7g significantly increased $\mathrm{p} 53$ expression (Fig. 3B and C). To examine the effect of p53 on the cell cycle, we performed flow cytometric analysis; however, no significant change in the cell cycle was detected after let7-g transfection (Fig. 3D).

Pre-let-7g miRNA negatively regulates protein expression of HMGA2 and K-Ras. Western blot analysis was performed in order to determine the effect of pre-let-7g on HMGA2 and $\mathrm{K}-\mathrm{Ras}$ protein expression levels. Our results showed that prelet-7g decreased protein levels of both K-Ras and HMGA2 (Fig. 4). Notably, knockdown of HMGA2 decreased the protein expression of K-Ras; however, knockdown of K-Ras did not alter the expression of HMGA2 (Fig. 4).
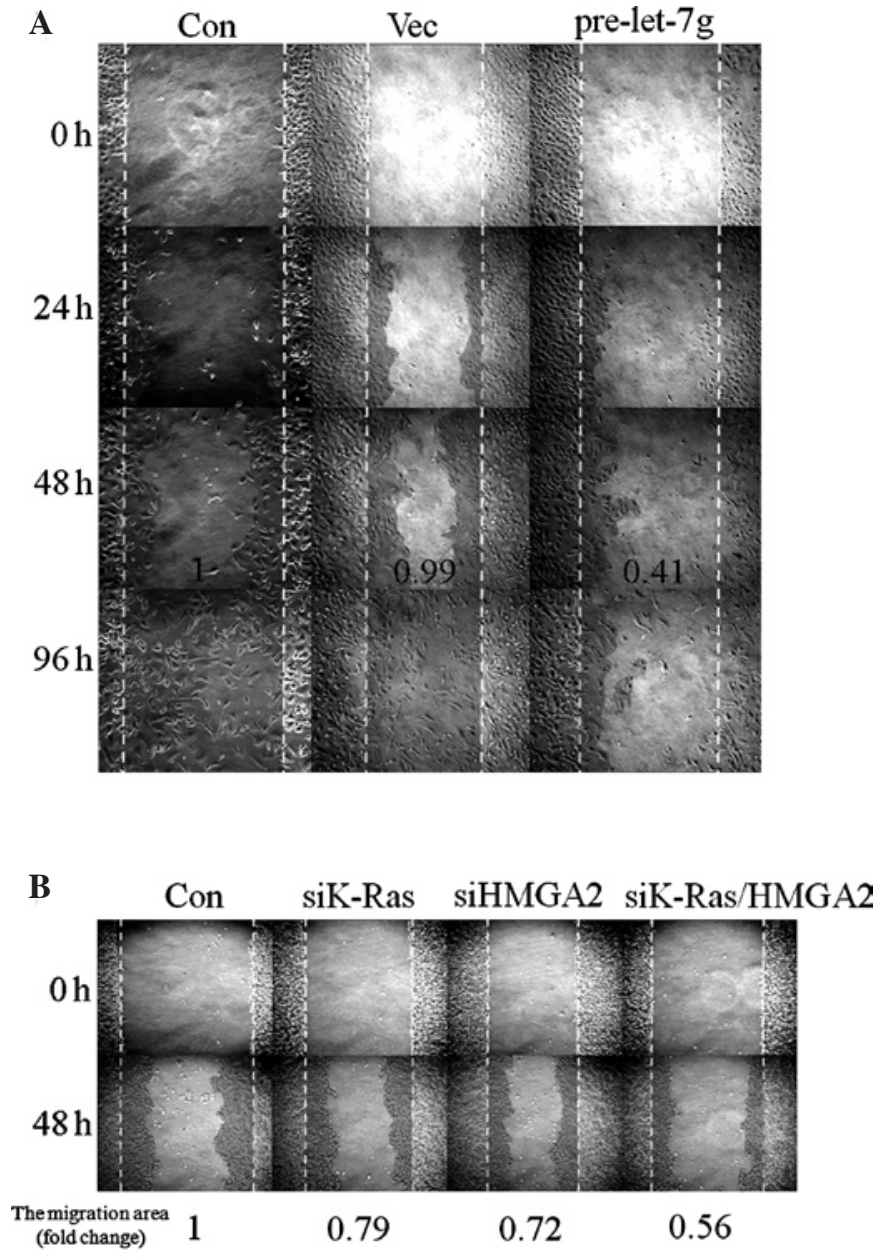

Figure 5. Inhibitory effect of pre-let-7g on cell migration. (A) Cell migration rate via wound healing observed in the A549 cells incubated for 0, 24, 48 and $96 \mathrm{~h}$ after transfection in the presence or absence of pre-let7-g and introduction of cell cycle S-phase inhibitor. (B) Identical to the protocol in A, cell migration rate via wound healing was observed in the A549 cells incubated for $48 \mathrm{~h}$ after transfection with siHMGA2 or siK-Ras, or co-transfection with siHMGA2/K-Ras compared with the A549 control cells, respectively. Con, control; Vec, vector control; siK-Ras, K-Ras siRNA; siHMGA2, HMGA2 siRNA, siK-Ras/HMGA2, co-transfected HMGA2 siRNA and K-Ras siRNA. Data represent the mean $\pm \operatorname{SEM}(n=3)$.

Pre-let-7g miRNA causes a strong inhibitory effect on cell migration. Since deficiency of let-7 miRNA causes poor prognosis (2), we hypothesized that deficient pre-let-7g might be associated with cancer cell migration. To determine the effect of let7-g on cell migration, a wound healing assay was performed after the blocking of the cell cycle with mitomycin C. Our result showed that pre-let-7g markedly inhibited cell migration after $48 \mathrm{~h}$ compared to the control (Fig. 5A). To examine the effect of K-Ras and HMGA2 on cell migration, we performed an additional wound healing assay in A549 cells transfected with K-Ras siRNA, HMGA2 siRNA, or co-transfected with both K-Ras/HMGA2 siRNAs at $48 \mathrm{~h}$ (Fig. 5B). Our results showed that down-regulation of two targets of pre-let7-g (K-Ras/HMGA2) significantly inhibited cell migration compared to each target alone (Fig. 5B).

Pre-let-7g miRNAs control E2F1 transcription factor and loss of E2F1 causes repression of cell migration. Recent studies 
A

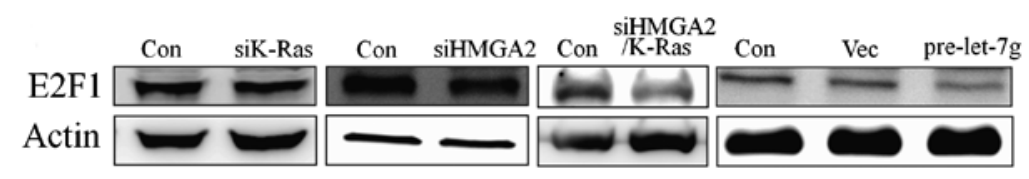

B
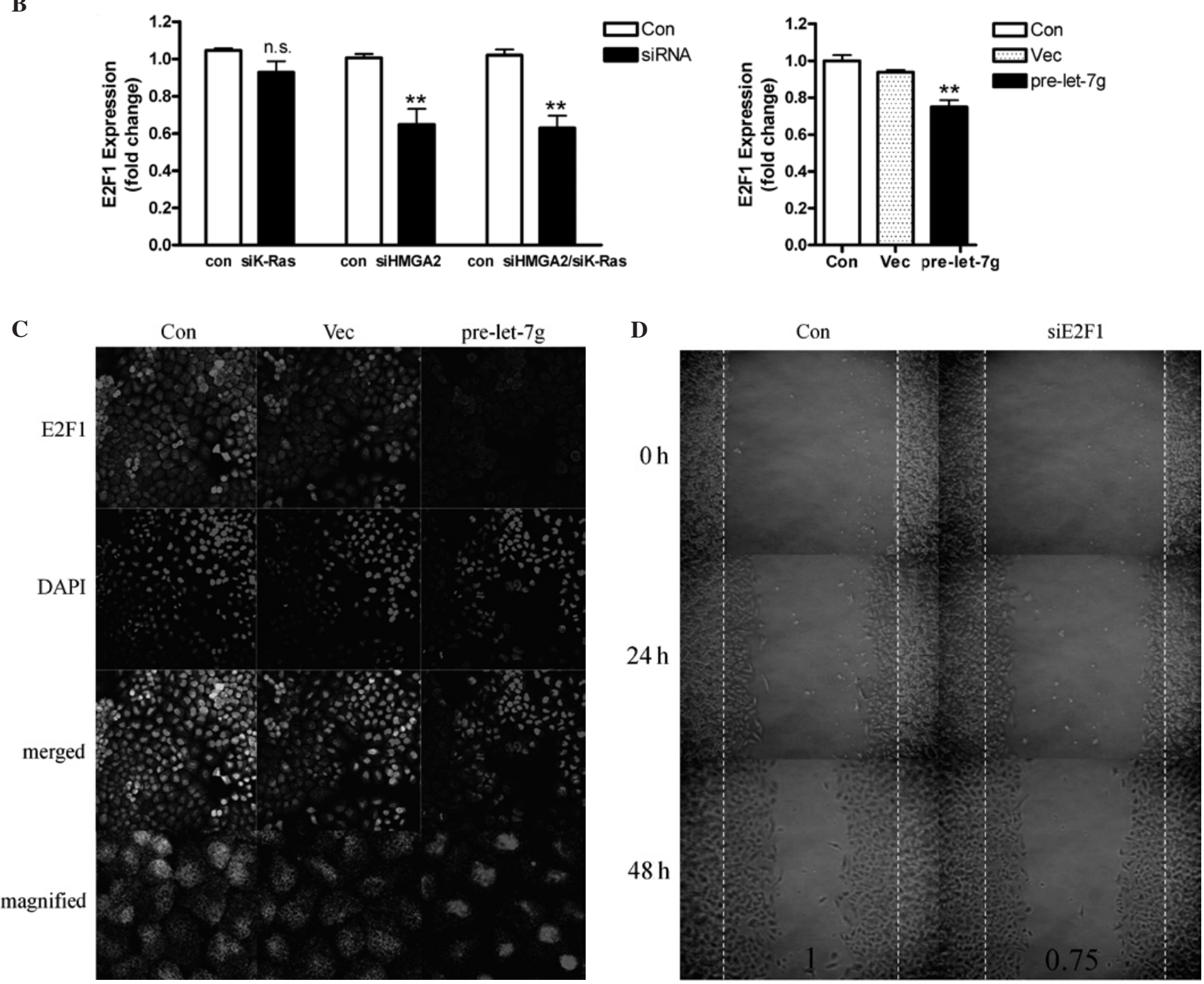

Figure 6. Inhibition of cell migration is associated with the down-regulation of E2F1 by pre-let-7g. (A) Representative Western blot analysis of the expression levels of E2F1 in the A549 cells transfected with let-7g or siHMGA2 or siK-Ras, or co-transfected with siHMGA2 and siK-Ras compared with the A549 control cells. (B) Quantification of expression data shown in A. (C) Immunofluorescence assay of E2F1 in the A549 cells transfected in the presence or absence of pre-let-7g. (D) Cell migration rate via wound healing was observed in the A549 cells incubated for 0,24 and $48 \mathrm{~h}$ after transfection in the presence or absence of siE2F1 and introduction of cell cycle S-phase inhibitor. Con, control; Vec, vector control; siK-Ras, K-Ras siRNA; siHMGA2, HMGA2 siRNA; siE2F1, E2F1 siRNA. Data represent the mean \pm SEM $(n=3) .{ }^{* *} \mathrm{P}<0.01$ statistically significant compared to the corresponding control.

have shown that overexpression of HMGA2 is associated with malignant tumors (14). HMGA2 has also been reported to facilitate the binding of E2F1 to promoters and to promote acetylation of histones on E2F1 target promoters (15). Based on the above findings, we hypothesized that E2F1 expression might be correlated with a change in cell migration by pre-let-7g. In order to examine whether or not pre-let-7g affects E2F1 expression, we performed Western blotting to analyze E2F1 expression in A549 cells after pre-let-7g transfection. Our results revealed that pre-let-7g negatively regulated E2F1 (Fig. 6A and B). Additionally, in order to investigate whether or not the down-regulation of E2F1 was triggered by pre-let-7g- mediated down-regulation of HMGA2, Western blotting was performed after siHMGA2 and/or siK-Ras transfection. Our results revealed that down-regulation of HMGA2 negatively regulated E2F1, whereas no such effects were observed for siK-Ras (Fig. 6B). To observe subcellular localization and expression of E2F1, we also performed immunochemistry. Fluorescence microscopic images revealed that pre-let-7g decreased the expression of E2F1 in the nucleus (Fig. 6C; top right). A wound healing assay was also performed to determine whether or not down-regulated E2F1 inhibits cell migration. Our results revealed that the down-regulation of E2F1 markedly repressed cell migration in A549 cells (Fig. 6D). 


\section{Discussion}

In the present study, pre-let-7g miRNA levels were reduced in lung cancer cell lines (Fig. 1). A decreased level of let-7g was previously found to be associated with poor clinical prognoses for lung cancer patients (2). In this study, we investigated the functional consequence of let-7g expression on lung cancer cell migration in order to study the potential correlation of let7-g with lung cancer metastasis. We used pre-let-7g, which has a stable hairpin structure and enough length for detection by RT-PCR compared to mature let-7g miRNA. Pre-let-7g recovered low let-7g expression in A549 lung carcinoma (Fig. 2A-E).

Notably, pre-let-7g increased $\mathrm{p} 53$ expression. The pre-let- $7 \mathrm{~g}$ level was also retained on a long-term basis. Together, increased p53 did not cause changes in the cell cycle or cell viability. Without doxycycline, let-7g alone was found to be insufficient to cause suppression of tumorigenesis (7). Doxycycline increases p53 levels and many miRNA transcripts expressed by p53 (16). Let-7g with doxycycline also increases the level of p53 (7). One recent study reported that the promoter for primary let $-7 \mathrm{~g}$ expression has a putative p53 binding site (13). Thus, a possible mechanism may be $\mathrm{p} 53$ regulation of the let- $7 \mathrm{~g}$ level.

To date, HMGA 2 and K-Ras, two targets of let-7g, have emerged from a number of studies $(7,10,17)$. As a target of let-7g, reduced HMGA2 has been demonstrated to inhibit aggressiveness in lung cancer (18-20). In particular, we focused on the combined targeting of K-Ras and HMGA2 by pre-let-7g. We also investigated the effects of pre-let7-g on cancer cell migration. Our results are consistent with results from a recent report indicating that the induction of K-Ras and HMGA2 caused features of metastasis (21). In the present study, the effect of the combined targeting of K-Ras and HMGA2 resulted in a stronger inhibition of cell migration compared to each target alone (Fig. 5B). Notably, gene silencing of HMGA2 led to decreased K-Ras, but not the silencing of K-Ras (Fig. 4B). Thus, according to this result, it is possible that HMGA2 may act as an upper signal of K-Ras.

Although the gene silencing efficiency of siHMGA2 is weaker than that of siK-Ras (Fig. 4A), the inhibition efficiency of siHMGA2 on cell migration was stronger (Fig. 5B). We subsequently confirmed that pre-let-7g reduced E2F1 expression (Fig. 6A and B). In particular, the E2F1 level was drastically decreased in the nucleus (Fig. 6C). These results are consistent with findings from several studies indicating that HMGA2 enhances E2F1 activity (15) and ablation of E2F1 impairs wound healing (22). Importantly, among the two targets of let-7g, the gene silencing of siHMGA2 significantly decreased E2F1 expression, while that of siK-Ras did not show significant change (Fig. 6A and B). Thus, according to these results, HMGA2 appears to modulate E2F1 activity, which suggests that let-7g-mediated E2F1 knockdown is correlated with inhibition of cancer cell migration (Fig. 6D).

Members of the let-7 family have been reported to regulate self-renewal $(23,24)$, proliferation $(25,26)$, the cell cycle $(17,27,28)$ and apoptosis $(29)$. However, most studies on let-7g have found a correlation with drug-sensitivity (7), radiation-sensitivity $(30,31)$ or metastasis $(32)$. It is possible that let-7g alone may not be a primary contributor to the suppression of tumorigenesis.
In summary, our data support the hypothesis that the recovery of pre-let-7g expression inhibits cell migration in lung cancer cells. Furthermore, pre-let-7g may be a powerful diagnostic marker for the aggressiveness of lung cancer cells. Taken together, our findings suggest that the use of pre-let- $7 \mathrm{~g}$ with therapeutic agents in the treatment of metastatic lung cancer may be clinically relevant.

\section{Acknowledgements}

This work was supported by grants from the National Research Foundation (\#2010-0000784) of the Ministry of Education, Science and Technology of Korea.

\section{References}

1. Bartel D: MicroRNA genomics, biogenesis, mechanism, and function. Cell 116: 281-297, 2004.

2. Takamizawa J, Konishi H, Yanagisawa K, et al: Reduced expression of the let-7 microRNAs in human lung cancers in association with shortened postoperative survival. Cancer Res 64: 3753-3756, 2004.

3. Filipowicz W, Bhattacharyya S and Sonenberg N: Mechanisms of post-transcriptional regulation by microRNAs: are the answers in sight? Nat Rev Genet 9: 102-114, 2008.

4. Bussing I, Slack F and Grosshans H: let-7 microRNAs in development, stem cells and cancer. Trends Mol Med 14: 400-409, 2008.

5. Lewis B, Burge C and Bartel D: Conserved seed pairing, often flanked by adenosines, indicates that thousands of human genes are microRNA targets. Cell 120: 15-20, 2005.

6. Lewis B, Shih I, Jones-Rhoades M, Bartel D and Burge C: Prediction of mammalian microRNA targets. Cell 115: 787-798, 2003.

7. Kumar M, Erkeland S, Pester R, et al: Suppression of non-small cell lung tumor development by the let-7 microRNA family. Proc Natl Acad Sci USA 105: 3903-3908, 2008.

8. Calin G, Sevignani C, Dumitru C, et al: Human microRNA genes are frequently located at fragile sites and genomic regions involved in cancers. Proc Natl Acad Sci USA 101: 2999-3004, 2004.

9. Johnson S, Grosshans H, Shingara J, et al: RAS is regulated by the let-7 microRNA family. Cell 120: 635-647, 2005.

10. Park S, Shell S, Radjabi A, et al: Let-7 prevents early cancer progression by suppressing expression of the embryonic gene HMGA2. Cell Cycle 6: 2585-2590, 2007.

11. Jiang J, Lee E, Gusev Y and Schmittgen T: Real-time expression profiling of microRNA precursors in human cancer cell lines. Nucleic Acids Res 33: 5394-5403, 2005.

12. Suzuki H, Yamagata K, Sugimoto K, Iwamoto T, Kato S and Miyazono K: Modulation of microRNA processing by 553 . Nature 460: 529-533, 2009.

13. Xi Y, Shalgi R, Fodstad O, Pilpel Y and Ju J: Differentially regulated micro-RNAs and actively translated messenger RNA transcripts by tumor suppressor $\mathrm{p} 53$ in colon cancer. Clin Cancer Res 12: 2014-2024, 2006.

14. Fedele M, Battista S, Manfioletti G, Croce C, Giancotti V and Fusco A: Role of the high mobility group A proteins in human lipomas. Carcinogenesis 22: 1583-1591, 2001.

15. Fedele M, Visone R, De Martino I, et al: HMGA2 induces pituitary tumorigenesis by enhancing E2F1 activity. Cancer Cell 9: 459-471, 2006.

16. Tarasov V, Jung P, Verdoodt B, et al: Differential regulation of microRNAs by 553 revealed by massively parallel sequencing. Cell Cycle 6: 1586-1593, 2007.

17. Johnson C, Esquela-Kerscher A, Stefani G, et al: The let-7 microRNA represses cell proliferation pathways in human cells. Cancer Res 67: 7713-7722, 2007.

18. Di Cello F, Hillion J, Hristov A, et al: HMGA2 participates in transformation in human lung cancer. Mol Cancer Res 6: 743-750, 2008.

19. Meyer B, Loeschke S, Schultze A, et al: HMGA2 overexpression in non-small cell lung cancer. Mol Carcinog 46: 503-511, 2007.

20. Sarhadi V, Wikman H, Salmenkivi K, et al: Increased expression of high mobility group A proteins in lung cancer. J Pathol 209: 206-212, 2006. 
21. Watanabe S, Ueda Y, Akaboshi S, Hino Y, Sekita Y and Nakao M: HMGA2 maintains oncogenic RAS-induced epithelial-mesenchymal transition in human pancreatic cancer cells. Am J Pathol 174: 854-868, 2009.

22. D'Souza S, Vespa A, Murkherjee S, Maher A, Pajak A and Dagnino L: E2F-1 is essential for normal epidermal wound repair. J Biol Chem 277: 10626-10632, 2002.

23. Yu F, Yao H, Zhu P, et al: let-7 regulates self renewal and tumorigenicity of breast cancer cells. Cell 131: 1109-1123, 2007.

24. Nishino J, Kim I, Chada K and Morrison S: Hmga2 promotes neural stem cell self-renewal in young but not old mice by reducing p16Ink4a and p19Arf expression. Cell 135: 227-239, 2008.

25. Esquela-Kerscher A, Trang P, Wiggins J, et al: The let-7 microRNA reduces tumor growth in mouse models of lung cancer. Cell Cycle 7: 759-764, 2008

26. Jakymiw A, Patel R, Deming N, et al: Overexpression of dicer as a result of reduced let-7 microRNA levels contributes to increased cell proliferation of oral cancer cells. Genes Chromosomes Cancer 49: 549-559, 2010.

27. Schultz J, Lorenz P, Gross G, Ibrahim S and Kunz M: MicroRNA let-7b targets important cell cycle molecules in malignant melanoma cells and interferes with anchorage-independent growth. Cell Res 18: 549-557, 2008
28. Legesse-Miller A, Elemento O, Pfau S, Forman J, Tavazoie S and Coller H: let-7 overexpression leads to an increased fraction of cells in $\mathrm{G} 2 / \mathrm{M}$, direct down-regulation of Cdc34, and stabilization of Weel kinase in primary fibroblasts. J Biol Chem 284: 6605-6609, 2009.

29. Shimizu S, Takehara T, Hikita $\mathrm{H}$, et al: The let-7 family of microRNAs inhibits Bcl-xL expression and potentiates sorafenibinduced apoptosis in human hepatocellular carcinoma. J Hepatol 52: $698-704,2010$

30. Jeong $\mathrm{S}$, Wu $\mathrm{H}$ and Park W: LIN28B confers radio-resistance through the posttranscriptional control of KRAS. Exp Mol Med 41: 912-918, 2009.

31. Wagner-Ecker M, Schwager C, Wirkner U, Abdollahi A and Huber P: MicroRNA expression after ionizing radiation in human endothelial cells. Radiat Oncol 5: 25-34, 2010.

32. Ji J, Zhao L, Budhu A, et al: Let-7g targets collagen type I (alpha) 2 and inhibits cell migration in hepatocellular carcinoma. J Hepatol 52: 690-697, 2010. 